\title{
Validation of the Comprehensive Arm Prosthesis and Rehabilitation Outcomes Questionnaire
}

\author{
Kerstin S. Baun', Nathan T. Kearns'², Jennifer K. Peterson', John M. Miguelez' \\ 'Clinical Services, Advanced Arm Dynamics, Redondo Beach, CA, USA \\ ${ }^{2}$ Department of Psychology, University of North Texas, Denton, TX, USA
}

Article Info

\section{Article Notes}

Received: July 27, 2021

Accepted: September 22, 2021

\section{*Correspondence:}

Jennifer K Peterson, MA PT, Advanced Arm Dynamics, 11671 Fountain Drive, Suite 220, Maple Grove, MN 55369, 763-4202767, USA; Email: jpeterson@armdynamics.com

${ }^{\circ} 2021$ Peterson JK. This article is distributed under the terms of the Creative Commons Attribution 4.0 International License.

\section{Keywords:}

Upper Limb Loss

Outcome Measure

Prosthesis, Satisfaction

Pain

Perceived Function

\section{Abstract}

Purpose: To develop and psychometrically evaluate the Comprehensive Arm Prosthesis and Rehabilitation Outcomes Questionnaire (CAPROQ), a 28-item, self-report measure of three key facets associated with successful rehabilitation (perceived function, satisfaction, and pain) designed specifically for the adult upper limb loss (ULL) population.

Materials and Methods: Using a national sample of adult ULL patients $(\mathrm{N}=240)$, factor structure, internal consistency, convergent/concurrent validity, and known group validity of the total CAPROQ score and three subscale scores were evaluated.

Results: Confirmatory factor analysis indicated adequate-to-strong factor loading on each subscale: satisfaction (.623-.913), perceived function (.572$.860)$ and pain (.422-.834). Internal consistencies for the total measure and measure subscales were good-to-excellent (.89-.95) and convergent validity indicated moderate-to-strong statistically significant associations between the CAPROQ subscales and relevant measures. Concurrent validity showed moderate associations between CAPROQ total score, prosthetic wear time, and psychosocial adjustment scores. Known group validity indicated significant differences on CAPROQ total score between initial and definitive fitting stages $(\mathrm{p}=.012)$.

Conclusion: Psychometric evaluation indicated that the CAPROQ and CAPROQ subscales were structurally sound, internally consistent, and demonstrated convergent validity with currently used assessments of perceived functioning, satisfaction, and pain. CAPROQ is needed for guiding individual patient care, improving care models and future prosthesis selection and development.

\section{List of abbreviations}

ACMC - Assessment of Capacity for Myoelectric Control

ADL - Activities of Daily Living

AM-ULA - Activities Measure for Upper Limb Amputees

BPI - Brief Pain Inventory

CAPPFUL - Capacity Assessment of Prosthetic Performance for the Upper Limb

CAPROQ - Comprehensive Arm Prosthesis and Rehabilitation Outcomes Questionnaire

CFA - Confirmatory Factor Analysis

DASH - Disability of the Arm, Shoulder, and Hand

FDA - Food and Drug Administration 
ICF - International Classification of Functioning, Disability and Health

OPUS-UEFS - Orthotic and Prosthetic User Survey Upper Extremity Functional Status

TAPES-R - Trinity Amputation and Prosthesis Experience Scales--Revised

UL - Upper limb

ULL- Upper limb loss

WIRB - Western Institutional Review Board

WHO - World Health Organization

\section{Introduction}

Improving treatment outcomes for the upper limb loss (ULL) community, including reducing prosthesis abandonment, hinge on the development and use of reliable, valid measures that comprehensively assess prosthetic rehabilitation, with strong consideration given to factors identified as central to success for this population, namely comfortable wear time and positive psychosocial adjustment ${ }^{1,2}$. The need for better outcome measures to guide clinical practice and product development for the ULL community is well-documented ${ }^{3-8}$. Although some of the assessments for prosthesis wearers are limited for use only for specific ULL subpopulations (e.g.., pediatric), others have insufficient evidence of psychometric validity, or simply do not address the unique needs of those with ULL, as they were created for broader upper limb (UL) injury populations ${ }^{5,6,9-11}$. This results in clinicians and researchers having limited options for evaluation to guide patient care and industry development, often forcing the utilization of measures not designed to address the distinctive issues of ULL and prosthesis function. Despite these known challenges, progress toward measure development has been modest $t^{5,12,13}$.

Generally, outcome measures for prosthesis users evaluate either performance with a prosthesis or prosthesis user's perceptions of factors related to their prosthesis and rehabilitation. Among others, the Assessment of Capacity for Myoelectric Control (ACMC) ${ }^{14}$, the Capacity Assessment of Prosthetic Performance for the Upper Limb (CAPPFUL) ${ }^{15,16}$, and the Activities Measure for Upper Limb Amputees (AMULA $)^{17}$ have been developed to assess performance. Patient report outcome measures provide a critical complement to performance measures by enabling insight into the individual's experience and perceptions. A few self-report outcome measures for adults who use UL prostheses have been reported in the literature; however, each has notable limitations that may undermine their utility within the ULL population. For example, the Orthotic and Prosthetic User Survey - Upper Extremity Functional Status (OPUS-UEFS) is a validated measure of prosthesis use and perceived function, evaluating the ease with which adults with ULL perform 28 activities of daily living and whether they use a prosthesis to perform each task ${ }^{18}$. However, prosthesis use is not incorporated into scoring and extant research has recommended item reduction, as well as need for further examination of validity ${ }^{5,19}$. In addition, the OPUSUEFS does not address critical factors linked to primary metrics of successful rehabilitation such as ability to wear prosthesis comfortably as needed, satisfaction with UL prosthetic rehabilitation and post amputation pain ${ }^{2,20}$. Finally, because the 28 tasks selected focus mostly on one handed activities, which can be completed with the intact hand only, the OPUS-UEFS does not necessarily detect perceived ability with a prosthesis or a change in skill with use of a prosthesis.

Other patient report measures with validation evidence for use in UL prosthetic rehabilitation include the Trinity Amputation and Prosthesis Experience Scales--Revised (TAPES-R) ${ }^{21-23}$, the Disabilities of Arm, Shoulder and Hand (DASH) questionnaire ${ }^{24-26}$, as well as the QuickDASH ${ }^{12,27}$. However, neither the DASH nor QuickDASH are designed specifically for the ULL and therefore do not capture information salient to successful prosthetic rehabilitation. The TAPES-R is an amputation/prosthesis-specific measure but was primarily designed for use with lower limb amputees. While it examines satisfaction, pain, and psychosocial adjustment, it lacks a singular metric of successful prosthetic rehabilitation. Importantly, all require further psychometric validation for the ULL population, including the need for assessment in larger, representative samples with adequate statistical power ${ }^{4,23,25,26}$.

\section{Comprehensive Arm Prosthesis and Rehabilitation Outcomes Questionnaire (CAPROQ) Background}

Research points to three critical facets of successful prosthetic rehabilitation outcomes: satisfaction with the device and rehabilitation, perception of functional abilities with that prosthesis, and post amputation pain, including pain associated with utilization of the device ${ }^{2,28,29}$. Further corroboration comes from a recent study conducted with over 800 veterans which found that among reasons for abandonment, notable issues were (1) lack of function, (2) factors related to satisfaction including cosmesis, lack of intuitive function, lack of reliability, "too much fuss" and (3) factors indicative of pain including comfort, weight and fatigue with use $^{30}$. Indeed, pain is very commonly reported post UL amputation $^{31}$, yet no measure specifically assesses pain in UL prosthesis users and specifically, the difference in pain when wearing and when not wearing a device. The Brief Pain Inventory (BPI) though extensively used has not been validated nor designed for this population and does not inform about the impact of prosthesis wear on pain ${ }^{32}$. This is an important distinction for the ULL 
community as wear and use of a prosthesis may in itself impact pain ${ }^{33}$.

The Comprehensive Arm Prosthesis and Rehabilitation Outcomes Questionnaire (CAPROQ) was developed to address these three key factors, providing both usable subscales and a single patient report metric of prosthetic rehabilitation outcomes based on subsection scores. More specifically, CAPROQ distinguishes itself in evaluating not only satisfaction with a prosthesis, but also satisfaction with broader elements of rehabilitation, perceived function in tasks that require the affected limb (unilateral and bilateral), and pain - both when wearing and when not wearing a prosthesis. The CAPROQ, therefore, improves upon existing measures in two primary ways. First, the CAPROQ is exclusively designed for the adult ULL community and empirical evaluation of psychometric properties (as described in this paper) is conducted with a nationwide, large, and representative sample from this community [demographics are listed in table 1]. Second, the CAPROQ is designed to comprehensively evaluate three empirically-derived domains related to successful rehabilitation outcomes (i.e., length of time the prosthesis

Table 1: Sociodemographic and Device-Related Characteristics of Sample

\begin{tabular}{|c|c|}
\hline Variables & Descriptive Information \\
\hline Age & $45.70 \pm 14.93$ \\
\hline \multicolumn{2}{|l|}{ Gender } \\
\hline Male & $160(66.7 \%)$ \\
\hline Female & $80(33.3 \%)$ \\
\hline \multicolumn{2}{|l|}{ Education $^{\mathrm{a}}$} \\
\hline Less than high school & $7(2.9 \%)$ \\
\hline High school & $41(17.1 \%)$ \\
\hline Some college & $55(22.9 \%)$ \\
\hline Associate degree & $26(10.8 \%)$ \\
\hline Bachelor degree & $58(24.2 \%)$ \\
\hline Postgraduate degree & $27(11.3 \%)$ \\
\hline \multicolumn{2}{|l|}{ Amputation Etiology } \\
\hline Trauma & $171(71.3 \%)$ \\
\hline Congenital & $47(19.6 \%)$ \\
\hline Disease & $12(5.0 \%)$ \\
\hline \multicolumn{2}{|l|}{ Years with Device } \\
\hline 5 years or less & $148(61.6 \%)$ \\
\hline $5-10$ years & $35(14.6 \%)$ \\
\hline $11-15$ years & $12(5.0 \%)$ \\
\hline $16-20$ years & $3(1.3 \%)$ \\
\hline 21 or more years & $42(17.6 \%)$ \\
\hline \multicolumn{2}{|l|}{ Amputation Side ${ }^{b}$} \\
\hline Right & $94(39.2 \%)$ \\
\hline Left & $110(45.8 \%)$ \\
\hline Both & $20(8.3 \%)$ \\
\hline Average device wear time (hours) & $7.79 \pm 4.89$ \\
\hline
\end{tabular}

Notes. Data presented as $\mathrm{n}(\%)$ and $\mathrm{M} \pm \mathrm{SD}$.

aData were missing for 8 participants (3.3\%).

${ }^{b}$ Data were missing for 16 participants (6.7\%). can be worn comfortably and positive psychosocial adjustment) : (1) satisfaction with their prosthesis and rehabilitation (e.g., comfort, appearance, knowledge of how to use it); (2) perceived function (e.g., ability to perform self-care tasks); and (3) pain both when wearing and when not wearing the prosthesis (e.g., residual limb pain, phantom limb pain). Description of all elements evaluated in these domains can be found in table 2 . The CAPROQ aims to comprehensively, yet concisely, evaluate these key patient-reported factors within a single measure to effectively guide individual patient care, assess care model effectiveness, and inform component selection and development for the ULL community. This initial psychometric study will describe the development of the measure and evaluate the factor structure, reliability (i.e., internal consistency), and validity (i.e., concurrent, known group) of the CAPROQ.

Table 2: CAPROQ Items and Factor Loadings from Confirmatory Factor Analysis

\begin{tabular}{|c|c|c|c|}
\hline \multirow[b]{2}{*}{ CAPROQ Items } & \multicolumn{3}{|c|}{ CAPROQ Subscales } \\
\hline & Satisfaction & $\begin{array}{l}\text { Perceived } \\
\text { Functioning }\end{array}$ & Pain \\
\hline \multicolumn{4}{|l|}{ Satisfied with... } \\
\hline ...comfort & .871 & & \\
\hline ...predictability & .839 & & \\
\hline ...appearance & .722 & & \\
\hline ...functionality & .904 & & \\
\hline ...suiting your lifestyle & .863 & & \\
\hline ...total & .913 & & \\
\hline ...knowledge of how to use & .623 & & \\
\hline ...effort required to operate & .824 & & \\
\hline \multicolumn{4}{|l|}{ How easily can you... } \\
\hline ...open a card size envelope & & .853 & \\
\hline ...open a bag of chips & & .791 & \\
\hline ...open a jar & & .794 & \\
\hline ...zip up a jacket & & .800 & \\
\hline ...remove a credit card & & .718 & \\
\hline ...put on a baseball cap & & .572 & \\
\hline ...place a box on shelf & & .712 & \\
\hline ...place a coat on hanger & & .860 & \\
\hline ...put on socks & & .743 & \\
\hline ...use dustpan and handbrush & & .775 & \\
\hline \multicolumn{4}{|l|}{ Amount of __ with device on ... } \\
\hline phantom pain & & & .442 \\
\hline residual limb pain & & & .436 \\
\hline numbness/pain in your back & & & .799 \\
\hline numbness/pain in your neck & & & .834 \\
\hline numbness/pain shoulder & & & .663 \\
\hline \multicolumn{4}{|l|}{ Amount of __ without device on... } \\
\hline phantom pain & & & .447 \\
\hline residual limb pain & & & .422 \\
\hline numbness/pain in your back & & & .769 \\
\hline numbness/pain in your neck & & & .823 \\
\hline numbness/pain shoulder & & & .685 \\
\hline
\end{tabular}




\section{Methods}

\section{Framework Conceptualization and Instrument Development}

CAPROQ addresses activity and participation domains as described in the WHO ICF ${ }^{34}$. It also assesses environmental and personal factors related to the assessed health condition (ULL). These additional factors are important to address in this population to obtain a holistic assessment of patient function. The CAPROQ was developed using a cyclical and iterative process as described in the Food and Drug Administration's (FDA) Guidance for Industry on patient reported outcome measures ${ }^{35}$. Patient input and evaluation of the measure throughout its development were critical to this process. Additional important considerations included method of administration, appropriateness of the recall period, response options, patient burden, and administrator burden. The latter two factors are especially salient, as time and healthcare resources must be efficiently and effectively allocated.

\section{Initial Development and Description of Domains}

Development of the CAPROQ began with literature review and a conceptual framework generated by subject matter experts (i.e., occupational and physical therapists, UL prosthetic specialists). The need for concise, quantifiable subsections in the areas of satisfaction, perceived function in activities of daily living (ADL), and pain were identified from the extant prosthetic rehabilitation literature ${ }^{2,28,29}$, and questions were subsequently generated by occupational and physical therapists specializing in UL amputation and prosthetic rehabilitation, as well as psychometric researchers, using simple and easy-tounderstand wording (i.e., $8^{\text {th }}$ grade reading level.)

\section{Revision of Measure}

The initial set of 86 questions were administered to a group of 43 ULL patient volunteers. A series of item reduction analyses (e.g., exploratory factor analysis [EFA], reliability analyses evaluating Cronbach's alpha) and an expert panel of occupational and physical therapists aided in narrowing of items to 8 satisfaction, 10 perceived functioning, and 10 pain questions for inclusion in the validation study of the CAPROQ. Item removal was based primarily on two factors: representativeness of items (from EFA) and utility of items. For example, for satisfaction questions, if items were identified as redundant (e.g., satisfaction with "color" and satisfaction with total "appearance"), the broader, more general question was retained. For perceived functioning, items that correlated most strongly with the overall construct were retained, while those showing weaker association were eliminated. For pain, items pertaining primarily to the trunk and affected side were retained.

\section{CAPROQ Overview and Scoring}

Satisfaction. The satisfaction questions ask about elements of the prosthesis and rehabilitation. Questions seek to explore patient's perceptions on prosthesis fit, function and esthetics; the prosthetic training they received; and their knowledge of how to operate their device. Sample items include, "How satisfied are you with the functionality of your prosthesis?" and "How satisfied are you with your knowledge of how to use your prosthesis?" Responses to eight items are given on a 10-point, Likert-type scale with descriptive anchors at 0 ("Very Dissatisfied") and 10 ("Very Satisfied"). Higher scores therefore indicate higher levels of overall satisfaction.

Perceived functioning. The perceived functioning questions ask participants about their perceived ability to perform primarily bimanual tasks in various functional planes of movement - at transverse body midline, shoulder height, and foot level. Respondents are asked to rank how easy it is to complete a task based on their performance using only their upper limbs (with or without prosthesis, depending on fitting stage) over the past 30 days. Sample items include, "open a card size envelope" and "zip up a jacket." Responses to 10 items are given on a 10-point, Likert-type scale with descriptive anchors at 0 ("Unable") and 10 ("Very Easy"). Higher scores therefore indicate higher levels of overall perceived functioning.

Pain. The pain questions ask participants about phantom and residual limb pain, as well as neck, back and shoulder pain. Each question asks for pain level (a) when wearing a prosthesis and (b) when not wearing a prosthesis. Sample items include, "phantom pain: pain that originates in the part of the limb that is no longer remaining" and "numbness or pain in your back". Responses to 10 items are given on a 10-point, Likert-type scale with descriptive anchors at 0 ("No Pain") and 10 ("Extreme Pain"). To align with the directionality of scoring of the satisfaction and perceived functioning subscales, scoring is weighted in reverse (e.g., $0=10,1=9$, etc.). Thus, after reverse scoring, higher scores indicate less pain.

Total CAPROQ score. A singular total CAPROQ score is derived by standardizing each of the CAPROQ subscales (i.e., for equal weighting) and summing these standardized scores together. Higher total scores on the CAPROQ indicate better rehabilitation outcomes and reflect the individual's perceived success with their prosthesis and rehabilitation; specifically, satisfaction with their prosthesis and rehabilitation, greater functional performance, and lesser pain.

\section{Procedures}

Participants were recruited through a combination of three methods: national email outreaches to persons with ULL from the researcher's client data base; small group 
and individual email outreach initiated by multiple site researchers/clinicians across the United States including Southwest, Northwest, Northeast and Midwest regions; and personal discussion at regularly scheduled prosthetic rehabilitation appointments with patients meeting study criteria. The study was approved by the Western Institutional Review Board (WIRB). Research began in July 2018 and concluded in March 2019. Participants had to meet the following inclusion criteria: have an UL amputation at any level, be at any stage of the prosthesis fitting process (initial fitting, definitive fitting, post definitive fitting), have any type of prosthesis (passive, body powered, electric, hybrid and/or activity specific), present with any type of amputation/limb loss etiology (disease, trauma, congenital), be able to understand study directions and content, and be between the ages of 18 and 95. Exclusion criteria included not having an amputation, not having a prosthesis, having any type of cognitive impairment that would preclude understanding of the study directions or content, being under age 18 or over 95. A total of 240 participants meeting provisional eligibility criteria were consecutively recruited and provided with the online survey consisting of the CAPROQ, the DASH, the Brief Pain Inventory Short Form (BPI), and the TAPES-R, presented sequentially in this order. More detailed descriptions of each measure can be found in Appendix A. Sections within the CAPROQ were also presented sequentially, however, questions within each CAPROQ subsection were presented in a randomized order. Of those that initiated the study, 210 individuals meeting inclusion criteria completed the CAPROQ 201 completed the DASH, 189 completed the BPI, and 169 completed the TAPES-R. Demographics describing the study population can be found in table 1 .

\section{Data Analytic Plan}

The following goals were established to psychometrically assess the structure, reliability, and validity of the CAPROQ: (1) evaluate the CAPROQ higher-order structural model with confirmatory factor analysis (CFA); (2) evaluate internal consistency of the three CAPROQ subscales and total measure; (3) evaluate concurrent validity via zero-order correlations between the CAPROQ subscales and relevant measure, as well as between the total CAPROQ score and relevant prosthetic rehabilitation outcomes; and (4) known-group validity based on evaluation of the associations between the total CAPROQ score and two stages of prosthetic fitting (i.e., initial and definitive).

Confirming Factor Structure. A CFA was fitted to confirm the 28-item, higher-order factor structure of the CAPROQ (i.e., item loading on three latent subscales, and those three latent subscales loading on singular, overarching CAPROQ factor). Given that measurement scales on each of the CAPROQ items comprised more than five categories, the data were considered intervally-scaled for analyses. According to $\mathrm{Hu}$ and Bentler ${ }^{36}$, models with excellent fit have the following fit statistics: CFI and TLI $\geq$ 0.95 (0.90-0.94) and RMSEA and SRMR $\leq 0.06$ (0.07-0.08). The parenthetical values listed above indicate adequate-togood model fit. Generally, RMSEA less than 0.06 indicate good fit, whereas RMSEA above that threshold, but less than 0.08 , indicates medium but acceptable fit ${ }^{37}$.

Reliability and Validity. Cronbach's alpha coefficients were used to assess the internal consistency of the CAPROQ subscales and CAPROQ total score. For convergent validity of the subscales, zero-order Pearson correlation coefficients were used to assess associations between the CAPROQ satisfaction subscale and the satisfaction subscales of the TAPES-R; the CAPROQ perceived disability subscale and the total score on the DASH; and the CAPROQ pain subscale and the five primary pain indices on the BPI. For concurrent validity of the total measure, zero-order Pearson correlation coefficient evaluated the association between the CAPROQ total score and wear time (hours of comfortable wear time), TAPES general and social adjustment and TAPES adjustment to limitations and activity restrictions subscales. For known-group validity, an independent-samples t-test evaluated differences between CAPROQ total scores at two distinct fitting stages (i.e., initial versus definitive).

Software and Data Management. Analyses of factor structure were conducted using $R$ (version 4.0.2) statistical software. Descriptive statistics, reliability, and validity analyses were conducted using SPSS (Version 26.0). Zero-order correlation coefficients $(r)$ and $p$-values were interpreted for significance and effect size in the convergent and concurrent validity analyses. Assumptions for each statistical analysis were checked and met (e.g., normality). Less than $0.03 \%$ of the data were missing. Therefore, maximum likelihood was used to handle missing data.

\section{Results}

\section{Confirming Factor Structure}

The CFA evidenced adequate-to-good fit to be retained as a reasonable model $(\mathrm{CFI}=.914 ; \mathrm{TFI}=.905$; $\mathrm{RMSEA}=$ .074 ; SRMR $=.068$ ). For the three CAPROQ subscales, all items indicated adequate-to-strong factor loading on each of the subscales: satisfaction (.623-.913); perceived functioning (.572-.860), and pain (.422-.834). Factor structure and loadings for the CFA model can be found in table 2. Correlations between subscales on the CAPROQ from the CFA are presented in table 3.

\section{Reliability and Validity}

Internal Consistency. Internal consistency was assessed using Cronbach's alpha for each of the three factors on the CAPROQ. Internal consistencies for the CAPROQ subscales 
Table 3: Correlation Matrix of CAPROQ Subscales

\begin{tabular}{|l|c|c|c|c|}
\hline & Satisfaction & $\begin{array}{c}\text { Perceived } \\
\text { Functioning }\end{array}$ & Pain & Cronbach's $\boldsymbol{\alpha}$ \\
\hline Satisfaction & - & & & .949 \\
\hline Perceived Functioning & .479 & - & & .932 \\
\hline Pain & -.175 & -.284 & - & .890 \\
\hline
\end{tabular}

were good-to-excellent, with alphas on the subscales ranging from .89 to .95 . Internal consistency of the total measure was excellent with an alpha of .92. See table 3 for full results of internal consistency coefficients.

Convergent Validity (Subscales). Results of the convergent validity analyses for the CAPROQ satisfaction subscale indicated moderate-to-strong statistically significant associations between the CAPROQ and all satisfaction subscales on the TAPES-R, including esthetic satisfaction $(r=.68)$ and functional satisfaction $(r=.80)$. Results of the convergent validity analyses for the CAPROQ perceived disability subscale indicated strong statistically significant association between the CAPROQ and the DASH total score $(r=-.61)$. Lastly, results of the convergent validity analyses for the CAPROQ pain subscale indicated strong statistically significant association between the CAPROQ and all pain-related indices on the BPI, including worst pain in the past 24 hours $(r=-.61)$, least pain in the past 24 hours ( $r=-.53)$, average overall pain $(r=-.63)$, current pain $(r=-.61)$ and perceived pain interference with daily functioning $(r=-.63)$.

Concurrent Validity (Total). Results of the concurrent validity analyses for the CAPROQ total score indicated a moderate, statistically significant association between the CAPROQ total score and how many hours per day patients reported comfortable wear $(r=.31)$. Results also indicated moderate-to-strong statistically significant associations between the CAPROQ total score and general adjustment $(r$ $=.28)$, social adjustment $(r=.27)$, adjustment to limitations $(r=.51)$, and perceived activity restrictions $(r=-.34)$ related to their prothesis.

Known-Group Validity (Total). Result of the knowngroup validity analyses indicated that patients in initial stage of fitting ( $M=68.08)$ score significantly lower in the CAPROQ than patients at the later, definitive stage of fitting $(M=78.94), t(45)=2.62, p=.012)$.

\section{Discussion}

The adult ULL community is a unique population for whom outcome measure development continues to be needed. The importance of appropriate device selection, fit and training in reducing device abandonment and improving rehabilitation outcomes, necessitates developing reliable and valid measures that capture comprehensive data to guide clinical care as well as industry research ${ }^{2}$. Considering the complexity in adequately predicting these critical rehabilitation outcomes (i.e., considerations relating to multiple domains in the WHO classification of function, disability, and health ${ }^{34}$, complete yet concise instruments are required to adequately capture patient experiences ${ }^{35}$. Currently available self-report measures are lacking in either comprehensiveness (i.e., the OPUS-UEFS designed to only assess perceived function and prosthesis use) or were intended for broader patient populations (i.e., the DASH designed for various UL musculoskeletal disorders, the TAPES-R designed primarily for lower limb amputations). Furthermore, validation studies of these self-report outcome measures lack sufficient sample size for strong empirical validation with the ULL population ${ }^{5,6,23,26}$. The CAPROQ having been studied with over 200 individuals nationwide with UL amputation, fills the need for a reliable and psychometrically valid, multi-faceted assessment that captures a comprehensive yet concise (i.e., 10 items or less for each subscale) reflection of the patient's perceived prosthetic and rehabilitation outcomes in a single metric.

Importantly, psychometric evaluation of the CAPROQ provides evidence for the higher-order (three-factor) structure of the measure; indicates good internal consistency across subscales; demonstrates good convergent validity between subscales and established measures of prosthetic satisfaction, perceived function, and several clinically relevant pain indices (e.g., general pain, pain interference). The total CAPROQ score demonstrates concurrent validity with the critical metric of rehabilitation success (i.e., prothesis-related wear time and psychosocial functioning); and indicates known-group validity between the total CAPROQ score and distinct prosthetic fitting stages.

There are two primary advantages of the CAPROQ over existing measures in the field. First, the CAPROQ was designed specifically for use among adults with ULL, regardless of the individual's prosthetic device fitting stage, UL prosthetic device(s) or level of UL amputation. Second, the CAPROQ is designed to assess three critical domains related to UL prosthetic use in a singular, comprehensive measure with easy-tounderstand questions and straight-forward scoring and interpretation of total scores. This information may be useful in highlighting areas that need improvement through prosthetic modification, prosthetic training, or therapeutic intervention. Furthermore, it may highlight strengths and weaknesses of various prostheses and prosthetic components for prescriptive decisions, provide prosthetic component justification and illuminate future development needs. 


\section{Limitations}

Findings from the current study should be considered in light of several limitations. First, although items with factor loadings greater than .40 are generally retained $38-40$, the phantom and residual limb pain items did have lesser factor loadings than the other items on the pain domain. Future work is needed to replicate and confirm the validity of the CAPROQ factor structure. Second, despite the CAPROQ being designed to evaluate patients prior to prosthesis fitting in areas of perceived function and pain when not wearing a prosthesis, validation of these subsections at this stage of care was not included in this validation study. Lastly, the current study consists entirely of cross-sectional data. As such, further psychometric evaluation utilizing larger samples and longitudinal designs is needed in order to establish testretest reliability, minimal detectable/clinical change, and, eventually, to establish norms on the CAPROQ for different levels of amputation.

\section{Conclusion}

The CAPROQ is a patient reported outcome measure designed specifically for the adult ULL community and can be administered throughout the continuum of care to assess patient perception of outcomes related to post amputation rehabilitation and prosthetic intervention. The CAPROQ is designed to balance comprehensiveness (i.e., evaluation of three domains empirically-related to UL rehabilitation outcomes - satisfaction with the prosthesis, perception of function, and pain when wearing and not wearing a prosthesis) and conciseness; indeed, patient and administrator burden is relatively low with average administration time requiring approximately 30 minutes. Initial psychometric evaluation indicates good-to-excellent internal consistency, convergent validity of CAPROQ subscales with other established measures, concurrent validity of the CAPROQ total score with other established measures of successful prosthetic rehabilitation and known group validity between stage of prosthetic fitting. As such, the CAPROQ may provide an excellent complement to performance-based measures (e.g., CAPPFUL ${ }^{15}$ ) for capturing a holistic assessment of patients presenting with ULL that may help improve patient outcomes: guide patient care, reduce device abandonment, inform future prosthesis selection and product development.

\section{Acknowledgments}

These data and findings have not been previous presented. This research did not receive any specific grant from funding agencies in the public, commercial, or notfor-profit sectors.

\section{Disclosure of Interest}

We certify that we have affiliations with or financial involvement (eg, employment, consultancies, honoraria, stock ownership or options, expert testimony, grants and patents received or pending, royalties) with an organization or entity with a financial interest in, or financial conflict with, the subject matter or materials discussed in the manuscript AND all such affiliations and involvements are disclosed. Nathan Kearns received personal fees from Advanced Arm Dynamics, both during the conduct of the study and outside the submitted work. Nathan Kearns was partially funded by the National Institute on Alcohol Abuse and Alcoholism (F31AA027142) and the National Institute on Drug Abuse (T32DA016184). These funding sources had no direct role/influence in the development, analysis, and/or dissemination of this paper. Kerstin Baun, Jennifer Peterson, John Miguelez are salaried employees of Advanced Arm Dynamics, outside the submitted work.

\section{References}

1. NiMhurchadha S, Gallagher P, MacLachlan M, Wegener ST. Identifying successful outcomes and important factors to consider in upper limb amputation rehabilitation: an international web-based Delphi survey. Disability and Rehabilitation. 2013;35(20):1726-33.

2. Biddiss E., Chau T, Upper-limb prosthetics: Critical factors in device abandonment. Am J Phys Med Rehabil. 2007; 86(12):977-87.

3. Wang S, Hsu CJ, Trent L, Ryan T, Kearns NT, Civillico EF, Kontson KL. Evaluation of performance-based outcome measures for the upper limb: a comprehensive narrative review. PM\&R. 2018 Sep 1;10(9):951-62.

4. Wright V. Prosthetic outcome measures for use with upper limb amputees: A systematic review of the peer-reviewed literature, 1970 to 2009. JPO: Journal of Prosthetics and Orthotics. 2009 Oct 1;21(9):P3-63.

5. Burger H, Franchignoni F, Heinemann AW, Kotnik S, Giordano A. Validation of the orthotics and prosthetics user survey upper extremity functional status module in people with unilateral upper limb amputation. Journal of rehabilitation medicine. 2008 May 5;40(5):393-9.

6. Resnik, L; Borgia, M. Reliability and validity of outcome measures for upper limb amputation. Journal of Prosthetics and Orthotics, 2012; 24:192-201.

7. Hill W, Kyberd P, Hermansson LN, Hubbard S, Stavdahl Ø, Swanson S. Upper limb prosthetic outcome measures (ULPOM): a working group and their findings. JPO: Journal of Prosthetics and Orthotics. 2009 Oct 1;21(9):P69-82.

8. Roach KE. Measurement of health outcomes: reliability, validity and responsiveness. JPO: Journal of Prosthetics and Orthotics. 2006 Jan 1;18(6):P8-12.

9. Hill W, Stavdahl $\emptyset$, Hermansson LN, Kyberd P, Swanson S, Hubbard S. Functional outcomes in the WHO-ICF model: establishment of the upper limb prosthetic outcome measures group. JPO: Journal of Prosthetics and Orthotics. 2009 Apr 1;21(2):115-9.

10. Hubbard S. Pediatric upper limb outcome measurement. JPO: Journal of Prosthetics and Orthotics. 2009 Oct 1;21(9):P64-8.

11. Miller LA, Swanson S. Summary and recommendations of the academy's state of the science conference on upper limb prosthetic outcome measures. JPO: Journal of Prosthetics and Orthotics. 2009 Oct 1;21(9):P83-9.

12. Resnik L, Borgia M. Reliability, validity, and responsiveness of the QuickDASH in patients with upper limb amputation. Archives of physical medicine and rehabilitation. 2015 Sep 1;96(9):1676-83. 
13. Resnik L, Borgia M, Silver B, Cancio J. Systematic review of measures of impairment and activity limitation for persons with upper limb trauma and amputation. Archives of physical medicine and rehabilitation. 2017 Sep 1;98(9):1863-92.

14. Hermansson LM, Fisher AG, Bernspång B, Eliasson AC. Assessment of capacity for myoelectric control: a new Rasch-built measure of prosthetic hand control. Journal of rehabilitation medicine. 2005;37(3):166-71.

15. Kearns NT, Peterson JK, Walters LS, Jackson WT, Miguelez JM, Ryan T. Development and Psychometric Validation of Capacity Assessment of Prosthetic Performance for the Upper Limb (CAPPFUL). Archives of physical medicine and rehabilitation. 2018 Sep 1;99(9):1789-97.

16. Boyle A, Prejean B, Ruhde L, Pool K, Bollinger C, Miguelez J, Conyers D, Ryan T, Kontson KL. Capacity Assessment of Prosthetic Performance for the Upper Limb (CAPPFUL): Characterization of Normative Kinematics and Performance. PM\&R. 2020 Jan 23.

17. Resnik L, Adams L, Borgia M, Delikat J, Disla R, Ebner C, Walters LS, Development and evaluation of the activities measure for upper limb amputees. Archives of physical medicine and rehabilitation. $2013 \mathrm{Mar}$ 1;94(3):488-94.

18. Heinemann AW, Bode RK, O’Reilly C. Development and measurement properties of the Orthotics and Prosthetics Users' Survey (OPUS): a comprehensive set of clinical outcome instruments. Prosthetics and Orthotics International. 2003, 27:191-206.

19. Jarl GM, Heinemann AW, Hermansson LMN. Validity evidence for a modified version of the Orthotics and Prosthetics Users' Survey. Disability and Rehabilitation: Assistive Technology. 2012; 7(6):469-478.

20. Jang CH, Yang HS, Yang HE, Lee SY, Kwon JW, Yun BD, Choi JY, Kim SN, Jeong HW. A survey on activities of daily living and occupations of upper extremity amputees. Annals of rehabilitation medicine. 2011 Dec;35(6):907.

21. Gallagher P, MacLachlan M. Development and psychometric evaluation of the Trinity Amputation and Prosthesis Experience Scales (TAPES). Rehabilitation Psychology. 2000 May;45(2):130.

22. Gallagher P, Franchignoni F, Giordano A, MacLachlan M. Trinity amputation and prosthesis experience scales: a psychometric assessment using classical test theory and rasch analysis. American journal of physical medicine \& rehabilitation. 2010 Jun 1;89(6):487-96.

23. Desmond DM, MacLachlan M. Factor structure of the Trinity Amputation and Prosthesis Experience Scales (TAPES) with individuals with acquired upper limb amputations. American journal of physical medicine \& rehabilitation. 2005 Jul 1;84(7):506-13.

24. Hudak PL, Amadio PC, Bombardier C, Beaton D, Cole D, Davis A, Hawker G, Katz JN, Makela M, Marx RG, Punnett L. Development of an upper extremity outcome measure: the DASH (disabilities of the arm, shoulder, and head). American journal of industrial medicine. 1996 Jun;29(6):602-8.

25. Østlie K, Franklin RJ, Skjeldal OH, Skrondal A, Magnus P. Assessing physical function in adult acquired major upper-limb amputees by combining the Disabilities of the Arm, Shoulder and Hand (DASH) Outcome Questionnaire and clinical examination. Archives of physical medicine and rehabilitation. 2011 Oct 1;92(10):1636-45.
26. Davidson J. A comparison of upper limb amputees and patients with upper limb injuries using the Disability of the Arm, Shoulder and Hand (DASH). Disabil Rehabil 2004;26:917-23.

27. Gummesson C, Ward MM, Atroshi I. The shortened disabilities of the arm, shoulder and hand questionnaire (Quick DASH): validity and reliability based on responses within the full-length DASH. BMC musculoskeletal disorders. 2006 Dec 1;7(1):44.

28. Yamamoto M, Chung KC, Sterbenz J, Shauver MJ, Tanaka H, Nakamura T, Oba J, Chin T, Hirata H. Cross-sectional International Multicenter Study on Quality of Life and Reasons for Abandonment of Upper Limb Prostheses. Plastic and Reconstructive Surgery Global Open. 2019 May; $7(5)$.

29. Ritchie S, Wiggins S, Sanford A. Perceptions of cosmesis and function in adults with upper limb prostheses: a systematic literature review. Prosthetics and orthotics international. 2011 Dec;35(4):332-41.

30. Resnik L, Ekerholm S, Borgia M, Clark MA. A national study of Veterans with major upper limb amputation: Survey methods, participants, and summary findings. PloS one. 2019;14(3).

31. Hanley MA, Ehde DM, Jensen M, Czerniecki J, Smith D, Robinson LR. Chronic Pain Associated with Upper-Limb Loss. Am. J Phys Med Rehabil. 2009; 88(9):742-779.

32. Keller S, Bann CM, Dodd SL, Schein J, Mendoza TR, Cleeland CS. Validity of the brief pain inventory for use in documenting the outcomes of patients with noncancer pain. The Clinical journal of pain. 2004 Sep 1;20(5):309-18.

33. Lotze M, Grodd W, Birbaumer N, Erb M, Huse E, Flor H. Does use of a myoelectric prosthesis prevent cortical reorganization and phantom limb pain? Nat Neurosci. 1999; 2(6):501-2.

34. World Health Organization. ICF training Beginner's Guide. 2002 [cited; Available from: http://www.who.int/classifications/icf/ training/icfbeginnersguide.pdf]

35. U.S. Department of Health and Human Services, Food and Drug Administration. Guidance for industry: patient-reported outcome measures: use in medical product development to support labeling claims: draft guidance. Health and Quality of Life Outcomes. 2009 Dec. https://www.fda.gov/downloads/drugs/guidances/ucm193282.pdf https://www.fda.gov/media/77832/download

36. Hu LT, Bentler PM. Cutoff criteria for fit indexes in covariance structure analysis: Conventional criteria versus new alternatives. Structural equation modeling: a multidisciplinary journal. 1999 Jan 1;6(1):1-55.

37. Browne MW, Cudeck R. Alternative ways of assessing model fit. Sociological methods \& research. 1992 Nov;21(2):230-58.

38. Li CH. Confirmatory factor analysis with ordinal data: Comparing robust maximum likelihood and diagonally weighted least squares. Behavior research methods. 2016 Sep 1;48(3):936-49.

39. Gunzler DD, Morris N. A tutorial on structural equation modeling for analysis of overlapping symptoms in co-occurring conditions using MPlus. Statistics in medicine. 2015 Oct 30;34(24):3246-80.

40. Manly BFJ. Factor analysis. In: Manly BFJ, ed. Multivariate statistical methods. A primer. London, United Kingdom: Chapman \& Hall, 1994:93-106. 


\section{Appendix A. Summary of Measures}

\section{Disabilities of Arm Shoulder and Hand Questionnaire (DASH):}

The DASH Outcome Measure is a 30-item, self-report questionnaire designed to measure physical function and symptoms in people with any of several musculoskeletal disorders of the UL. The questionnaire was designed to help describe the disability experienced by people with UL disorders and also to monitor changes in symptoms and function over time. Testing has shown that the DASH performs well in both these roles. The total results are reported on $0-100$ scales with higher scores indicating greater perceived disability.

\section{Trinity Amputation and Prosthetic Experience Scales-Revised (TAPES-R):}

Trinity Amputation and Prosthesis Experience Scale--Revised was developed to assess the adaptation to lower limb amputation and the psychosocial processes involved in adjusting to prosthetic use. Designed for post-prosthesis delivery, the TAPES-R is a self-administered questionnaire that asks the participant about different aspects of wearing a prosthesis including their general adjustment, social adjustment, adjustment to limitations, activity level and aesthetic and functional satisfaction with the prosthesis. Additionally, it addresses phantom and residual limb pain and other medical problems unrelated to the amputation ${ }^{21}$.

\section{Orthotic and Prosthetic User Survey-Upper Extremity Functional Status:}

This patient report measure originally consisting of 28 activities of daily living. Individuals rank each task's difficulty as either "very easy", "easy", "slightly difficult", "very difficult", "cannot perform" or "not applicable". In addition, respondents are asked to indicate whether they are using or not using their prosthesis to complete each task ${ }^{18,19}$.

\section{Brief Pain Inventory (BPI):}

Initially designed to assess pain caused by cancer, the BPI has since been used for a wide range of conditions. Two domains-pain intensity (severity) and pain interference (the impact pain has on function) have been recommended for inclusion in all chronic pain trials. Items are rated on a 0-10 scale.

\section{Capacity Assessment of Prosthesis Performance of the Upper Limb (CAPPFUL):}

An 11-item performance-based outcome measure that assesses the prosthesis user's control skill, component utilization, compensatory movements, maladaptive and adaptive, as well as task completion and time for task completion. Total scoring based on $0-100 \%$ with $100 \%$ being equal to unimpaired sound hand function, reflects the prosthesis user's ability to replicate function of the sound hand.

\section{Activities Measure for Upper Limb Amputees (AM-ULA):}

A performance-based measure for adults with UL amputation, consisting of 18 items and factoring task completion, speed, movement quality, skillfulness of prosthesis use and independence into its rating system. Scores range from 0-40 with higher scores reflecting higher function.

\section{Assessment of Capacity for Myoelectric Control (ACMC):}

A validated 22 item observational measure examining need for external support, grip force, coordination of both hands, different positions and in motion, repetitive grasp and release and the need for visual feedback when using a myoelectric prosthesis. The ACMC requires specialized training to administer. 